\title{
Theory of the Star Formation Rate
}

\author{
Paolo Padoan ${ }^{1}$ and Åke Nordlund ${ }^{2}$ \\ ${ }^{1}$ ICREA \& ICC, University of Barcelona, Marti i Franquès 1, E-08028 Barcelona, Spain \\ email: ppadoan@icc.ub.edu \\ ${ }^{2}$ Niels Bohr Institute, University of Copenhagen, Juliane Maries Vej 30, DK-2100, \\ Copenhagen, Denmark \\ email: aake@nbi.dk
}

\begin{abstract}
This work presents a new physical model of the star formation rate (SFR), tested with a large set of numerical simulations of driven, supersonic, self-gravitating, magneto-hydrodynamic (MHD) turbulence, where collapsing cores are captured with accreting sink particles. The model depends on the relative importance of gravitational, turbulent, magnetic, and thermal energies, expressed through the virial parameter, $\alpha_{\mathrm{vir}}$, the rms sonic Mach number, $\mathcal{M}_{\mathrm{S}, 0}$, and the ratio of mean gas pressure to mean magnetic pressure, $\beta_{0}$. The SFR is predicted to decrease with increasing $\alpha_{\mathrm{vir}}$ (stronger turbulence relative to gravity), and to depend weakly on $\mathcal{M}_{\mathrm{S}, 0}$ and $\beta_{0}$, for values typical of star forming regions $\left(\mathcal{M}_{\mathrm{S}, 0} \approx 4-20\right.$ and $\left.\beta_{0} \approx 1-20\right)$. The star-formation simulations used to test the model result in an approximately constant SFR, after an initial transient phase. Both the value of the SFR and its dependence on the virial parameter found in the simulations agree very well with the theoretical predictions.
\end{abstract}

Keywords. ISM: kinematics and dynamics, MHD, stars: formation, turbulence

\section{Introduction}

The star-formation process is slow. Only a small fraction of the mass of cold gas is converted into stars in a free-fall time, $\tau_{\mathrm{ff}}$, both on Galactic scale (Zuckerman \& Palmer 1974; Williams \& McKee 1997) and on the scale of individual clouds (Krumholz \& Tan 2007, Evans et al. 2009). Several authors have proposed that the observed supersonic turbulence may be the reason for the low star formation rate (SFR). The turbulence is responsible for much of the complex and filamentary density structure observed in molecular clouds, and prestellar cores are likely assembled as the densest regions in this turbulent fragmentation process (Padoan et al. 2001). But even if supersonic turbulence intermittently creates dense regions that are gravitationally unstable, it does it inefficiently; its net effect on the large scale is to suppress star formation when the total turbulent kinetic energy exceeds the total gravitational energy.

Due to the importance of turbulence, the SFR depends primarily on the ratio of the turbulent kinetic energy, $E_{\mathrm{K}}$, and the gravitational energy, $E_{\mathrm{G}}$, of a star-forming region. This ratio is expressed by the virial parameter introduced by Bertoldi \& McKee (1992),

$$
\alpha_{\mathrm{vir}} \sim \frac{2 E_{\mathrm{K}}}{E_{\mathrm{G}}}=\frac{5 \sigma_{\mathrm{v}, 1 \mathrm{D}}^{2} R}{G M},
$$

where $\sigma_{\mathrm{v}, 1 \mathrm{D}}$ is the one-dimensional $\mathrm{rms}$ velocity, $R$ and $M$ the cloud radius and mass respectively, and $G$ the gravitational constant, and it has been assumed the cloud is a sphere with uniform density.

Krumholz \& McKee (2005) derived a theoretical model where the SFR is primarily controlled by the virial parameter. In this model, it is assumed that the gas mass above some critical density, $\rho_{\mathrm{cr}}$, is gravitationally unstable, and the fraction of this unstable 
mass is computed assuming the gas density obeys a Log-Normal pdf (Nordlund \& Padoan 1999). The idea of relying on the density pdf was also exploited in Padoan \& Nordlund (2002) and in Padoan \& Nordlund (2004) to explain the stellar IMF and the origin of brown dwarfs, and by Padoan (1995) to model the SFR.

The model of Krumholz \& McKee (2005) did not include magnetic fields and was calibrated and tested using low-resolution SPH simulations by Vazquez-Semadeni et al. (2003). In this work we propose a new model of the SFR that includes magnetic fields and that is tested with an unprecedented set of large numerical simulations of driven, supersonic, self-gravitating, MHD turbulence, where collapsing cores are represented by accreting sink particles. To model the process of star formation we must include gravitational, turbulent, magnetic, and thermal energies. Their relative importance is expressed by the virial parameter, $\alpha_{\mathrm{vir}}$, the rms sonic Mach number, $\mathcal{M}_{\mathrm{S}, 0}$, and the mean gas pressure to mean magnetic pressure, $\beta_{0}$, and we derive a model that depends explicitly on all three non-dimensional parameters. In the non-magnetized limit of $\beta_{0} \rightarrow \infty$, our definition of the critical density for star formation has the same dependence on $\alpha_{\text {vir }}$ as in the model of Krumholz \& McKee (2005), but our derivation does not rely on the concepts of local turbulent pressure support and sonic scale.

\section{Critical density in MHD Turbulence}

Including both thermal and magnetic pressures, the pressure balance condition for magneto-hydrodynamic (MHD) shocks is:

$$
\rho_{\mathrm{MHD}}\left(c_{\mathrm{S}}^{2}+v_{\mathrm{A}}^{2} / 2\right)=\rho_{0}\left(v_{0} / 2\right)^{2},
$$

where $c_{\mathrm{S}}$ is the sound speed, $\rho_{0}$ and $\rho_{\mathrm{MHD}}$ the preshock and postshock gas densities, $v_{0} / 2$ the shock velocity, and $v_{\mathrm{A}}$ is the Alfvén velocity in the postshock gas defined by the postshock magnetic field perpendicular to the direction of compression. Because the field is amplified only in the direction perpendicular to the compression, the postshock perpendicular field is comparable to the total postshock field, and we can write, $v_{\mathrm{A}} \approx$ $B /(4 \pi \rho)^{1 / 2}$, where $B$ is the postshock magnetic field and $\rho$ the postshock gas density. The characteristic gas density and thickness of postshock layers are thus given by:

$$
\begin{gathered}
\rho_{\mathrm{MHD}}=\rho_{0}\left(\mathcal{M}_{\mathrm{S}, 0}^{2} / 4\right)\left(1+\beta^{-1}\right)^{-1}, \\
\lambda_{\mathrm{MHD}}=\left(\theta L_{0}\right)\left(\mathcal{M}_{\mathrm{S}, 0}^{2} / 4\right)^{-1}\left(1+\beta^{-1}\right),
\end{gathered}
$$

where $L_{0}$ is the size (e.g. the diameter for a sphere) of the system, $\theta L_{0}$, with $\theta \leqslant 1$, is the turbulence integral scale, and we have introduced the ratio of gas to magnetic pressure in the postshock gas, $\beta=2 c_{\mathrm{S}}^{2} / v_{\mathrm{A}}^{2}$. In the limit of $\beta \rightarrow \infty$, these expressions reduce to the corresponding HD ones.

In numerical simulations of supersonic and super-Alfvénic turbulence, it is found that, although $v_{\mathrm{A}}$ has a very large scatter for any given density, its mean value is nearly density independent, corresponding to a mean relation approaching $B \propto \rho^{1 / 2}$ for a very weak mean magnetic field (Padoan \& Nordlund 1999). In the specific MHD simulation used in this work, the mean value of $v_{\mathrm{A}}$ is almost exactly constant for any density $\rho \gtrsim 2 \rho_{0}$. Zeeman splitting measurements of the magnetic field strength in molecular cloud cores are also consistent with an average value of $v_{\mathrm{A}}$ nearly independent of density (Crutcher 1999).

We define the critical density as the density above which a uniform sphere of radius $\lambda_{\mathrm{MHD}} / 2$ is gravitationally unstable. To account for both thermal and magnetic support, we adopt the approximation of the critical mass for collapse, $M_{\mathrm{cr}}$, introduced by McKee 
(1989),

$$
M_{\mathrm{cr}} \approx M_{\mathrm{BE}}+M_{\phi}
$$

where $M_{\phi}$ is the magnetic critical mass for a sphere of radius $R$, mean density equal to the postshock density $\rho$, and constant mass-to-flux ratio,

$$
M_{\phi}=0.17 \pi R^{2} B / G^{1 / 2}=0.387 v_{\mathrm{A}}^{3} /\left(G^{3 / 2} \rho^{1 / 2}\right)
$$

where the numerical coefficient 0.17 is from Tomisaka et al. (1988) (see also Nakano \& Nakamura (1978) for the case of an infinite sheet, and McKee \& Ostriker 2007 for a discussion of ellipsoidal clouds and other geometries), and $M_{\mathrm{BE}}$ is the Bonnor-Ebert mass (Bonnor 1956; Ebert 1957) with external density equal to the postshock density $\rho$,

$$
M_{\mathrm{BE}}=1.182 c_{\mathrm{S}}^{3} /\left(G^{3 / 2} \rho^{1 / 2}\right)
$$

The critical density is then defined by the condition,

$$
M_{\mathrm{MHD}}\left(\rho_{\mathrm{cr}, \mathrm{MHD}}\right)=M_{\mathrm{BE}}\left(\rho_{\mathrm{cr}, \mathrm{MHD}}\right)+M_{\phi}\left(\rho_{\mathrm{cr}, \mathrm{MHD}}\right),
$$

where $M_{\mathrm{MHD}}(\rho)=(4 / 3) \pi \lambda_{\mathrm{MHD}}^{3} \rho$. Equation (2.7) results in the following expression for the critical density as a function of the three non-dimensional parameters, $\alpha_{\mathrm{vir}}, \mathcal{M}_{\mathrm{S}, 0}$, and $\beta$ :

$$
\frac{\rho_{\mathrm{cr}, \mathrm{MHD}}}{\rho_{0}}=0.067 \theta^{-2} \alpha_{\mathrm{vir}} \mathcal{M}_{\mathrm{S}, 0}^{2} \frac{\left(1+0.925 \beta^{-\frac{3}{2}}\right)^{\frac{2}{3}}}{\left(1+\beta^{-1}\right)^{2}},
$$

Based on numerical simulations, we adopt the value of $\beta=0.39$ for $\mathcal{M}_{\mathrm{S}, 0} \approx 10$ and $0.2 \lesssim \beta_{0} \lesssim 20$ (see details in Padoan \& Nordlund 2010, in preparation). The compilations of $\mathrm{OH}$ and $\mathrm{CN}$ Zeeman measurements by Troland \& Crutcher (2008) and Falgarone et al. (2008) give an average value of $\beta=0.34$ and 0.28 respectively, very close to the value adopted here.

\section{Gas Density PDF and Star Formation Rate}

We estimate the gas mass fraction that is turned into stars by computing the mass fraction above the critical density, as in Krumholz \& McKee (2005). For given values of $\alpha_{\mathrm{vir}}, \mathcal{M}_{\mathrm{S}, 0}$, and $\beta$ (or $\beta_{0}$ ), the critical density is fixed, and the mass fraction above the critical density is determined by the density pdf. In the HD case, the density pdf is known to be Log-Normal, with a standard deviation depending on the rms Mach number. We assume that the pdf can be approximated by a Log-Normal also in the MHD case,

$$
p_{\mathrm{MHD}}(x) d x=\frac{x^{-1}}{\left(2 \pi \sigma_{\mathrm{MHD}}^{2}\right)^{1 / 2}} \exp \left[-\frac{\left(\ln x+\sigma_{\mathrm{MHD}}^{2} / 2\right)^{2}}{2 \sigma_{\mathrm{MHD}}^{2}}\right] d x
$$

with

corresponding to

$$
\sigma_{x, \mathrm{MHD}} \approx\left(1+\beta^{-1}\right)^{-1 / 2} \mathcal{M}_{\mathrm{S}, 0} / 2
$$

$$
\sigma_{\mathrm{MHD}}^{2} \approx \ln \left[1+\left(\frac{\mathcal{M}_{\mathrm{S}, 0}}{2}\right)^{2}\left(1+\beta^{-1}\right)^{-1}\right] .
$$

In the non-magnetized case $(\beta=\infty)$, these equations reduce to the result of Padoan et al. (1997).

Assuming that a fraction $\epsilon$ of the mass fraction above the critical density is turned into stars in a free-fall time of the critical density, $\tau_{\mathrm{ff}, \mathrm{cr}}=\left(3 \pi /\left(32 G \rho_{\mathrm{cr}, \mathrm{MHD}}\right)\right)^{1 / 2}$, the star 
formation rate per free-fall time (the mass fraction turned into stars in a free-fall time) is given by

$$
\mathrm{SFR}_{\mathrm{ff}}=\epsilon \frac{\tau_{\mathrm{ff}, 0}}{\tau_{\mathrm{ff}, \mathrm{cr}}} \int_{x_{\mathrm{cr}}}^{\infty} x p_{\mathrm{MHD}}(x) d x=\epsilon \frac{x_{\mathrm{cr}}^{1 / 2}}{2}\left(1+\operatorname{erf}\left[\frac{\sigma^{2}-2 \ln \left(x_{\mathrm{cr}}\right)}{2^{3 / 2} \sigma}\right]\right)
$$

where $\tau_{\mathrm{ff}, 0}=\left(3 \pi /\left(32 G \rho_{0}\right)\right)^{1 / 2}$ is the free-fall time of the mean density, $x_{\mathrm{cr}}=\rho_{\mathrm{cr}, \mathrm{MHD}} / \rho_{0}$ given by equation (2.8), $\sigma=\sigma_{\mathrm{MHD}}$ given by equation (3.3), and the expression is valid also in the HD limit of $\beta \rightarrow \infty$. The value of $\epsilon$ is 1 in the HD case and 0.5 in the MHD case (see details in Padoan \& Nordlund 2010, in preparation).

\section{SFR in Simulations of Driven MHD Turbulence}

In order to test the SFR model, we have run a set of simulations of driven supersonic turbulence, on meshes with $500^{3}-1,000^{3}$ computational zones. Using the same methods and setup as in Padoan \& Nordlund (2002) and Padoan \& Nordlund (2004), we adopt periodic boundary conditions, isothermal equation of state, and random forcing in Fourier space at wavenumbers $1 \leqslant k \leqslant 2$ ( $k=1$ corresponds to the computational box size). The simulations are based on two initial snapshots of fully developed turbulence, one for HD and one for MHD. These snapshots are obtained by running the HD and the MHD simulations from initial states with uniform initial density and magnetic field, and random initial velocity field with power only at wavenumbers $1 \leqslant k \leqslant 2$, for approximately 5 dynamical times, on meshes with $1,000^{3}$ computational zones, with the driving force keeping the rms sonic Mach number at the approximate value of $\mathcal{M}_{\mathrm{S}, 0}=\sigma_{\mathrm{v}, 3 \mathrm{D}} / c_{\mathrm{S}} \approx 9$ (except for one $\mathrm{HD}$ run with $\mathcal{M}_{\mathrm{S}, 0} \approx 4.5$ ). The initial pressure ratio is $\beta_{0}=22.2$ in all MHD runs, and $\beta_{0}=\infty$ in the HD runs.

The star formation simulations start when the gravitational force is included. The computational mesh is downsized from $1,000^{3}$ to $500^{3}$ zones for the $500^{3}$ runs, or kept the same for the $1,000^{3}$ runs. The driving force is still active during the star-formation phase of the simulations, in order to achieve a stationary value of $\alpha_{\text {vir }}$ to correlate with the SFR. The virial parameter varies in the range $\alpha_{\mathrm{vir}}=0.22-2.04$. To define the virial parameter of the simulations, we have chosen to use equation (1.1), with $R=L_{0} / 2$, where $L_{0}$ is the box size, and $M$ equal to the total mass in the box, $M_{0}$. The virial parameter is then $\alpha_{\text {vir }}=5 v_{0}^{2} L_{0} /\left(6 G M_{0}\right)$, where $v_{0}$ is the three-dimensional rms velocity in the box. A collapsing region is captured by the creation of an accreting sink particle if the density exceeds a certain density threshold $\left(8,000\right.$ times the mean density in both $500^{3}$ and $1000^{3}$ runs). Further accretion (defined as density exceeding the density threshold) is collected onto the nearest sink particle if the distance is less than four grid zones. Sink particles are never merged. An example of a projected density field from a star formation simulation is shown in Figure 1.

\section{Models versus Numerical Results}

Figure 2 compares the SFR model with the numerical results. The HD simulations follow almost exactly the theoretical prediction with $\epsilon=1$, suggesting that all the gas with density above the critical value collapses in a timescale of order $\tau_{\mathrm{ff}, \mathrm{cr}}$, as assumed in the model. The dependence of $\mathrm{SFR}_{\mathrm{ff}}$ on $\alpha_{\mathrm{vir}}$ is too shallow to be consistent with the parametrization in Krumholz \& McKee (2005). Only the run with the highest $\alpha_{\text {vir }}$ deviates significantly $(\approx 50 \%)$ from the theoretical prediction. This is probably only a numerical resolution effect, because the corresponding higher resolution run yields a higher 


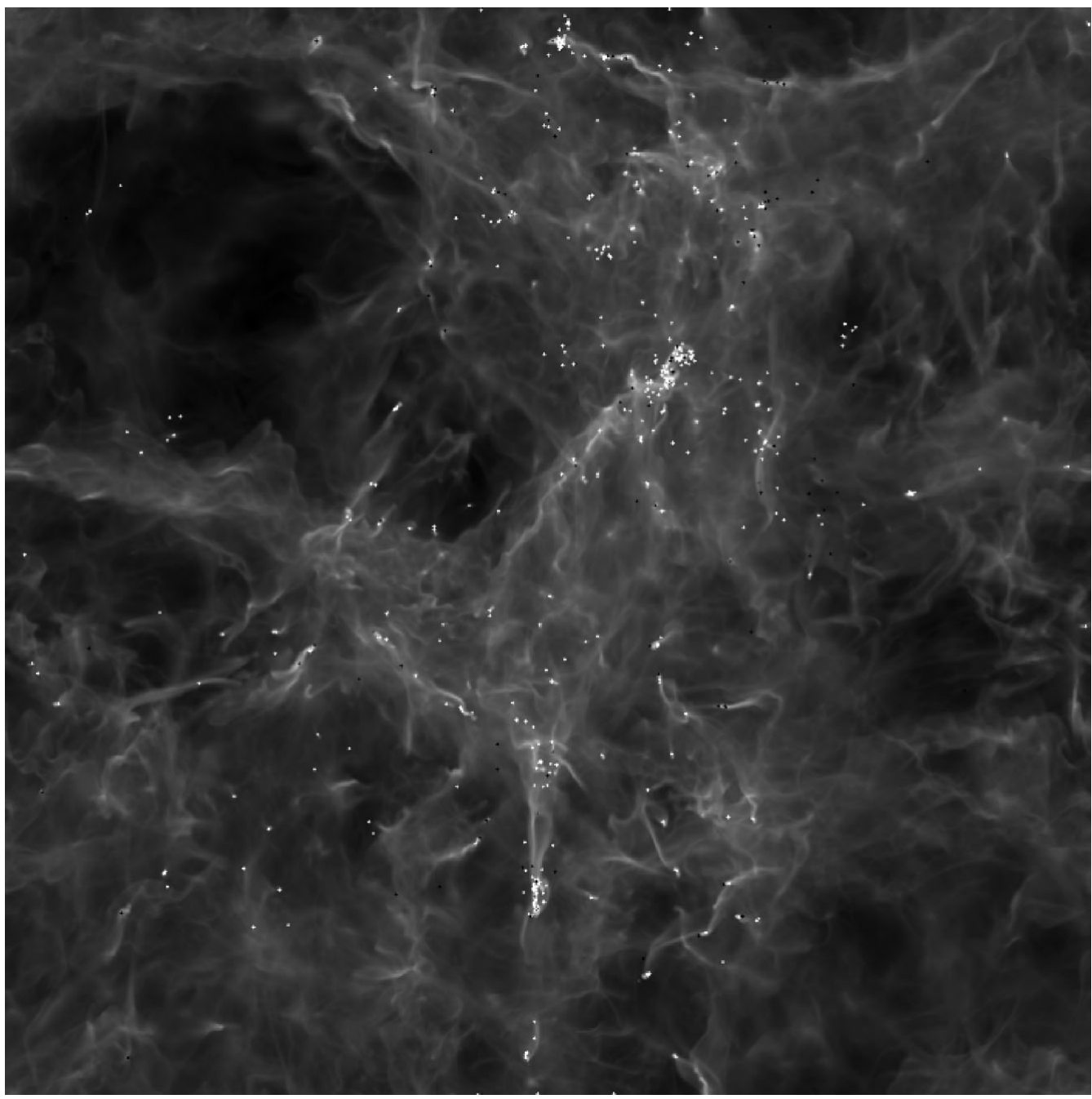

Figure 1. Logarithm of projected density from a snapshot of an exploratory $1,000^{3}$ run with $\beta_{0}=22.2, \mathcal{M}_{\mathrm{S}, 0}=18$, and $\alpha_{\mathrm{vir}}=0.9$, at a time when approximately $10 \%$ of the mass has been converted into stars. Bright dots show the positions of the stars (sink particles), while black dots are for brown dwarfs (some of which are still accreting and may later grow to stellar masses).

value of $\mathrm{SFR}_{\mathrm{ff}}$, nearly identical to the theoretical prediction. At $\alpha_{\mathrm{vir}}=0.95$, instead, the $500^{3}$ run is already converged to the SFR of the corresponding $1,000^{3}$ run (HD5 and HD8 respectively). The HD runs also confirm the theoretical prediction that $\mathrm{SFR}_{\mathrm{ff}}$ should increase with increasing $\mathcal{M}_{\mathrm{S}, 0}$ (the opposite of the prediction in Krumholz \& McKee (2005), as shown by the comparison of the runs with $\mathcal{M}_{\mathrm{S}, 0}=4.5$ and 9 , respectively. The lower Mach number run fits very well the theoretical prediction, confirming our choice of $\tau_{\mathrm{ff}, \mathrm{cr}}$ for the timescale of star formation.

There is good agreement between the MHD simulations and the theoretical model with $\epsilon=0.5$ as well, though the model predicts a significantly higher SFR than the $500^{3}$ simulation with the largest value of $\alpha_{\mathrm{vir}}$. This discrepancy may be entirely due to the insufficient numerical resolution of the simulation, because the $1,000^{3}$ run with the same virial parameter, $\alpha_{\mathrm{vir}}=2.04$, yields a value of $\mathrm{SFR}_{\mathrm{ff}}$ almost identical to the theoretical prediction. Like in the HD simulations, the case with $\alpha_{\text {vir }}=0.95$ seems to be already 


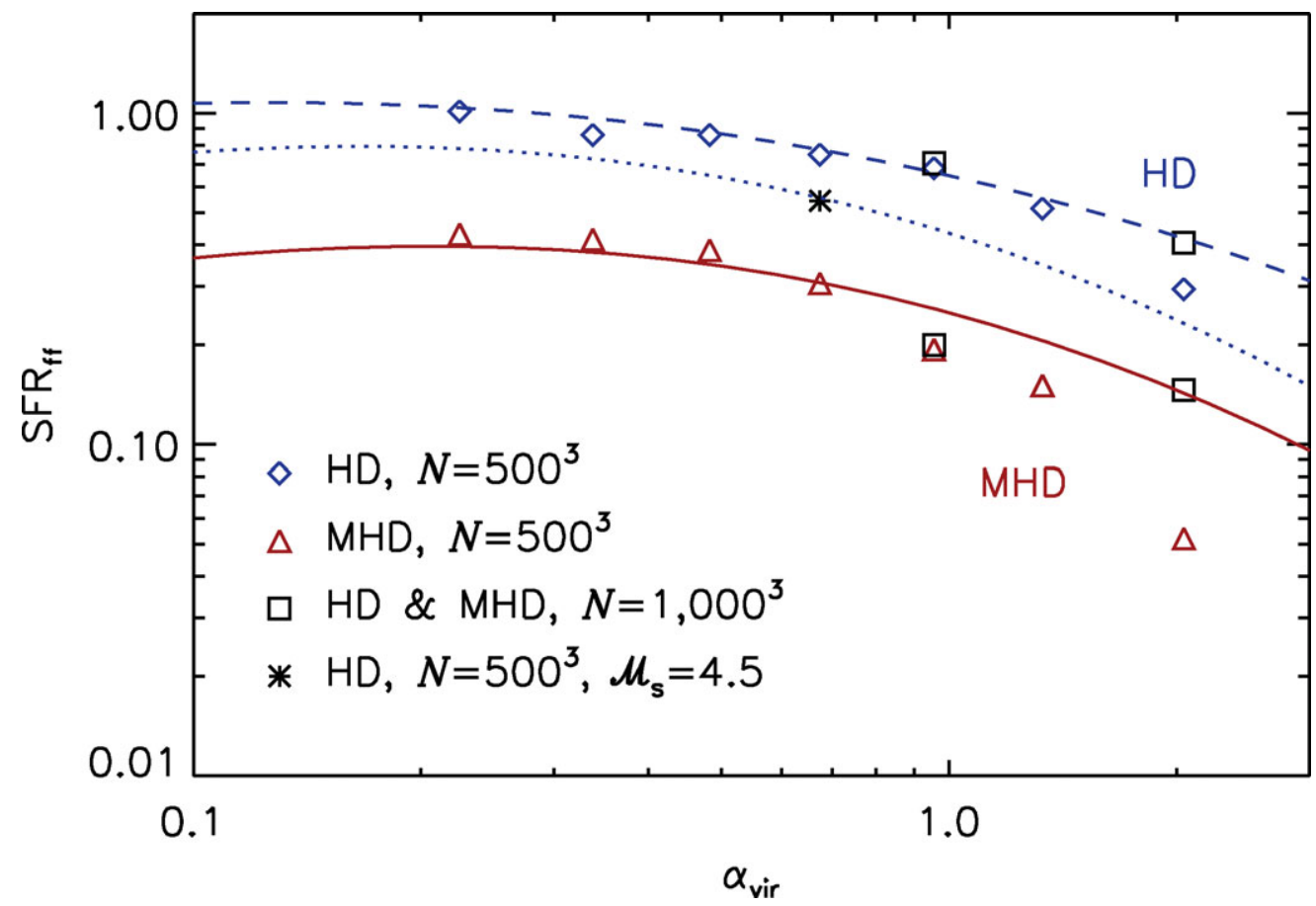

Figure 2. Star formation rate per free-fall time versus virial parameter for the $500^{3}$ MHD simulations (triangles) and for the $500^{3} \mathrm{HD}$ simulations (diamonds) with $\mathcal{M}_{\mathrm{S}, 0}=9$. The squares are for the $1,000^{3}$ runs, and the asterisk for the $500^{3} \mathrm{HD}$ run with $\mathcal{M}_{\mathrm{S}, 0}=4.5$. The MHD model with $\mathcal{M}_{\mathrm{S}, 0}=9, \beta_{0}=22.2$, and $b=0.63$ is shown by the solid line. The HD model $\left(\beta_{0}=\infty\right)$ is shown by the dashed line for $\mathcal{M}_{\mathrm{S}, 0}=9$, and by the dotted line for $\mathcal{M}_{\mathrm{S}, 0}=4.5$.

converged at a resolution of $500^{3}$ computational zones, as its $\mathrm{SFR}_{\mathrm{ff}}$ is nearly identical to that of the corresponding $1,000^{3}$ run (and only approximately $20 \%$ below the predicted value).

\section{Comparison with SFR in Molecular Clouds}

Evans et al. (2009) have estimated values of $\mathrm{SFR}_{\mathrm{ff}}$ in giant molecular clouds (GMCs) and within some of the dense cloud cores. They find values of $\mathrm{SFR}_{\mathrm{ff}}=0.03$ to 0.06 for GMCs with mean densities distributed around a mean value of $\langle n\rangle=390 \mathrm{~cm}^{-3}$, and $\mathrm{SFR}_{\mathrm{ff}}=0.05$ to 0.25 for dense cores with mean densities 50-200 times those of the GMCs. These values are computed by assuming that all the stars detected (by their infrared excess) have been formed in the last 2 Myr. The authors report a best estimate of $2 \pm 1$ Myr for the lifetime of the Class II phase, so the $\mathrm{SFR}_{\mathrm{ff}}$ could be $50 \%$ lower, or $100 \%$ higher than the values given above. Accounting for this uncertainty, one gets $\mathrm{SFR}_{\mathrm{ff}}=0.02$ to 0.12 for GMCs, and $\mathrm{SFR}_{\mathrm{ff}}=0.03$ to 0.5 for dense cores, suggesting a characteristic value of order 0.1 .

For a range of values of $\mathcal{M}_{\mathrm{S}, 0}$ characteristic of $\mathrm{MCs}$, we predict $\mathrm{SFR}_{\mathrm{ff}} \approx 0.12$ to 0.28 at $\alpha_{\text {vir }}=2$. These values should be reduced by a factor of two or three (Matzner \& McKee 2000; André et al. 2010), to account for mass loss from stellar outflows and jets, not included in the model and in the simulations. With this reduction, our results are consistent with the relatively high values of $\mathrm{SFR}_{\mathrm{ff}}$ found by Evans et al. (2009). The 
definition of the virial ratio for our periodic box (Eq. 1.1) and for observed star forming regions is an important source of uncertainty. Heyer et al. (2009) have recently studied a large subset of the GMCs sample of Solomon et al. (1987). For each cloud, they compute LTE masses based on the $\mathrm{J}=1-0$ emission lines of ${ }^{13} \mathrm{CO}$ and ${ }^{12} \mathrm{CO}$. They find masses smaller by a factor of 2 to 5 than the virial masses derived by Solomon et al. (1987). Their revised velocity dispersion are also somewhat smaller than in Solomon et al. (1987), but their resulting virial parameters are still a factor of approximately 2-3 larger, with a mean value of $\alpha_{\mathrm{vir}}=2.8 \pm 2.4$. If the LTE-derived mass underestimates the real mass by a factor up to two, as argued by the authors, then the values of $\alpha_{\text {vir }}$ should be reduced by a factor of two. The mean value is therefore likely to lie in the range $\alpha_{\text {vir }}=1.4$ to 2.8 , with a very large scatter. If GMCs have a characteristic value of $\alpha_{\mathrm{vir}} \approx 2$, as suggested by this observational sample, the SFR predicted by our model for a reasonable range of values of $\mathcal{M}_{\mathrm{S}, 0}$, and accounting for a factor of two or three reduction due to mass-loss in outflows and jets, is then consistent with the recent observational estimates by Evans et al. (2009).

\section{Summary and Concluding Remarks}

This work presents a new physical model of the SFR that could be implemented in galaxy formation simulations. The model depends on the relative importance of gravitational, turbulent, magnetic, and thermal energies, expressed through the virial parameter, $\alpha_{\mathrm{vir}}$, the rms sonic Mach number, $\mathcal{M}_{\mathrm{S}, 0}$, and the ratio of the mean gas pressure to mean magnetic pressure, $\beta_{0}$. The value of $\mathrm{SFR}_{\mathrm{ff}}$ is predicted to decrease with increasing $\alpha_{\text {vir }}$, and to increase with increasing $\mathcal{M}_{\mathrm{S}, 0}$, for values typical of star forming regions $\left(\mathcal{M}_{\mathrm{S}, 0} \approx 4-20\right.$. In the complete absence of a magnetic field, $\mathrm{SFR}_{\mathrm{ff}}$ increases typically by a factor of three, proving the importance of magnetic fields in star formation, even when they are relatively weak (super-Alfvénic turbulence). The model predictions have been tested with an unprecedented set of large numerical simulations of supersonic MHD turbulence, including the effect of self-gravity, and capturing collapsing cores as accreting sink particles. The SFR in the simulations follow closely the theoretical predictions.

This work illustrates how the turbulence controls the SFR. It does not address how the turbulence is driven to a specific value of $\alpha_{\mathrm{vir}}$. Because much of the turbulence driving is likely due to SN explosions, the turbulent kinetic energy and the value of $\alpha_{\text {vir }}$ are coupled to the SFR in a feedback loop. The feedback determines the equilibrium level of the SFR (and hence also the equilibrium level of $\alpha_{\text {vir }}$ ) at large scales. If $\alpha_{\text {vir }}$ were to decrease (increase) relative to the equilibrium, the SFR would increase (decrease), according to the results of this work, resulting in an increased (decreased) energy injection rate by SN explosions, thus restoring a higher (lower) value of $\alpha_{\mathrm{vir}}$. The dependence of the SFR on $\alpha_{\text {vir }}$ found in this work suggests that this self-regulation may work quite effectively.

Cosmological simulations of galaxy formation provide the rate of gas cooling and infall, which sets the gas reservoir for the star formation process and thus ultimately controls the SFR. They also include prescriptions for the star formation feedback, known to be essential to recover observed properties of galaxies (Gnedin et al. 2009; Gnedin et al. 2010). Future galaxy formation simulations should adopt a physical SFR law with an explicit dependence on $\alpha_{\mathrm{vir}}, \mathcal{M}_{\mathrm{S}, 0}$, and $\beta$ as derived in this work, in order to correctly reflect specific conditions of protogalaxies at different redshifts. This requires a treatment of the star formation feedback capable of providing an estimate of $\alpha_{\text {vir }}$ on scales of order 10-100 pc, not far from the spatial resolution currently achieved by the largest cosmological simulations of galaxy formation. 


\section{Acknowledgements}

This research was supported in part by the NSF grant AST-0908740, and a grant from the Danish Natural Science Research Council. We utilized computing resources provided by the San Diego Supercomputer Center, by the NASA High End Computing Program, and by the Danish Center for Scientific Computing.

\section{References}

André, P., et al. 2010, arXiv:1005.2618

Beetz, C., Schwarz, C., Dreher, J., \& Grauer, R. 2008, Physics Letters A, 372, 3037

Bertoldi, F. \& McKee, C. F. 1992, ApJ, 395, 140

Bonnor, W. B. 1956, MNRAS, 116, 351

Brunt, C. M., Federrath, C., \& Price, D. J. 2010, MNRAS, 403, 1507

Crutcher, R. M. 1999, ApJ, 520, 706

Ebert, R. 1957, Zeitschrift fur Astrophysik, 42, 263

Elmegreen, B. G. 2000, ApJ, 530, 277

Elmegreen, B. G. 2007, ApJ, 668, 1064

Evans, N. J., et al. 2009, ApJ, 181, 321

Falgarone, E., Troland, T. H., Crutcher, R. M., \& Paubert, G. 2008, A\&SA, 487, 247

Federrath, C., Klessen, R. S., \& Schmidt, W. 2008, ApJL, 688, L79

Gnedin, N. Y., \& Kravtsov, A. V. 2010, ApJ, 714, 287

Gnedin, N. Y., Tassis, K., \& Kravtsov, A. V. 2009, ApJ, 697, 55

Heyer, M., Krawczyk, C., Duval, J., \& Jackson, J. M. 2009, ApJ, 699, 1092

Kritsuk, A. G., Norman, M. L., Padoan, P., \& Wagner, R. 2007, ApJ, 665, 416

Kritsuk, A. G., Ustyugov, S. D., Norman, M. L., \& Padoan, P. 2009, Journal of Physics Conference Series, 180, 012020

Kritsuk, A. G., Ustyugov, S. D., Norman, M. L., \& Padoan, P. 2009b, arXiv:0912.0546

Krumholz, M. R. \& McKee, C. F. 2005, ApJ, 630, 250

Krumholz, M. R. \& Tan, J. C. 2007, textitApJ, 654, 304

Li, P. S., Norman, M. L., Mac Low, M.-M., \& Heitsch, F. 2004, ApJ, 605, 800

Matzner, C. D. \& McKee, C. F. 2000, ApJ, 545, 364

McKee, C. F. 1989, ApJ, 345, 782

McKee, C. F. \& Ostriker, E. C. 2007, ARAA, 45, 565

Nakano, T. \& Nakamura, T. 1978, PASJ, 30, 671

Nordlund, Å. \& Padoan, P. 1999, in: J. Franco \& A. Carramiñana (eds.), Interstellar Turbulence (Cambridge University Press), p. 218

Padoan, P. 1995, MNRAS, 277, 377

Padoan, P., Juvela, M., Goodman, A. A., \& Nordlund, Å. 2001, ApJ, 553, 227

Padoan, P. \& Nordlund, A. 1999, ApJ, 526, 279

Padoan, P. \& Nordlund, Å. 2002, ApJ, 576, 870

-. 2004, ApJ, 617, 559

Padoan, P., Nordlund, Å., \& Jones, B. 1997, MNRAS, 288, 145

Solomon, P. M., Rivolo, A. R., Barrett, J., \& Yahil, A. 1987, ApJ, 319, 730

Tomisaka, K., Ikeuchi, S., \& Nakamura, T. 1988, ApJ, 335, 239

Troland, T. H. \& Crutcher, R. M. 2008, ApJ, 680, 457

Vazquez-Semadeni, E. 1994, ApJ, 423, 681

Vázquez-Semadeni, E., Ballesteros-Paredes, J., \& Klessen, R. S. 2003, ApJL, 585, L131

Vázquez-Semadeni, E., Kim, J., \& Ballesteros-Paredes, J. 2005, ApJL, 630, L49

Williams, J. P. \& McKee, C. F. 1997, ApJ, 476, 166

Zuckerman, B. \& Palmer, P. 1974, ARAA, 12, 279 